\title{
Especificações mínimas aceitáveis de um analisador de desfibriladores e cardioversores
}

\author{
José Carlos Teixeira de Barros Moraes*, Conrado Almeida de Oliveira
}

Resumo Esta pesquisa objetiva recomendar especificações que os analisadores de desfibriladores e cardioversores devam apresentar, tais como: erro máximo de medição, resolução, condições de operação e recursos desejáveis. As recomendações apresentadas baseiam-se nos quesitos demandados para a realização dos ensaios previstos pelas normas NBR IEC 60601-2-4:2005 e ANSI/AAMI DF80:2003. Uma avaliação das especificações fornecidas de quatro analisadores importados (Fluke QED6, Fluke Impulse 7000, MTK-Biomed Delta 3000 MKII e NETECH Delta 3000) e dois analisadores nacionais (Transmai JM-01 e Ourox JAU-100) também foi realizada, tendo encontrado diversas falhas de especificações em todos os analisadores. Estas falhas vão desde o não fornecimento de dados a condições aquém das desejáveis para um analisador de desfibriladores e cardioversores. Os problemas mais graves encontrados estão relacionados à não apresentação de especificações tais como precisão, exatidão de medição, erro máximo de medição ou outra informação que por sua vez auxiliaria na determinação do intervalo de abrangência para a medida, como a de energia pelo analisador Transmai JM-01, algo inaceitável para um instrumento de medição, incertezas elevadas e condições de operação aquém das necessárias para a realização dos ensaios determinados pelas normas NBR IEC 60601-2-4:2005 e ANSI/AAMI DF80:2003.

Palavras-chave Especificações, Analisador, Desfibrilador, Cardioversor.

\section{Minimum acceptable specifications for a defibrillator and cardioverter analyzer}

\begin{abstract}
This study aims to recommend specifications for defibrillator and cardioverter analyzers, such as maximum permissible measurement error, resolution, operating conditions and desirable features. The recommendations are based on the test requirements from the ABNT NBR IEC 60601-2-4:2005 and ANSI/AAMI DF80:2003 standards. An evaluation of the specifications provided by four imported analyzers (Fluke QED 6, Fluke Impulse 7000, MTK-Biomed Delta 3000 MKII and NETECH Delta 3000) and two Brazilian analyzers (Transmai JM-01 and Ourox JAU-110) has also been performed, and several problems were found with specifications of those analyzers. These problems range from unsupplied data to less than desirable conditions for a defibrillator and cardioverter analyzer. The most serious problems encountered are related to the lack of specifications such as precision, accuracy, maximum permissible measurement error or other specifications that would help determine the coverage interval of the measure, an unacceptable mistake for a measurement instrument. Other problems were related to measurement error and operating conditions not complying with the requirements of the ABNT NBR IEC 60601-2-4:2005 and the ANSI/AAMI DF80:2003 standards.
\end{abstract}

Keywords Specifications, Analyzer, Defibrillator, Cardioverter. 


\section{Extended Abstract}

\section{Introduction}

Defibrillators and cardioverters perform a fundamental role in clinical applications, both medical and veterinarian. Therefore, the correct and careful definition of their specifications is mandatory to ensure both safety and essential performance for this category of medical electrical equipment.

The analysis of defibrillators and cardioverters, as well as preventive and corrective maintenance activities, can be experimentally performed with the use of a device called defibrillator and cardioverter analyzer.

This article proposes to contribute in determining the minimum specifications for defibrillator and cardioverter analyzers that are in compliance with both local and international technical standards and regulations. This study defines a so called minimum specification that is desirable for a defibrillator and cardioverter analyzer and also checks if analyzers currently available in the market comply with such prescriptions and if their specifications are presented appropriately to the user.

\section{Materials and Methods}

\section{Basic specifications for a defibrillator and cardioverter analyzer}

In order for a defibrillator analyzer to be capable to perform the tests required in the ABNT NBR IEC 60601-2-4:2005 (Associação..., 2005a) and the ANSI/AAMI DF80:2003 (American..., 2003) standards under all foreseen operation conditions, it must be able to perform measurements under all environmental conditions foreseen for defibrillators and cardioverters in those standards, since they will be under the same conditions during testing.

The ANSI/AAMI DF80:2003 (American..., 2003) standard requires that all testing instruments used in the tests specified in it should have the highest possible accuracy and a tolerance margin of no more than a third of the tolerance for the variable being measured, or $\pm 5 \%$ of the variable being measured for which only maximum and minimum limits are provided. Such values are in accordance to those considered desirable for a measurement instrument and follow the guidelines in NBR ISO 10012-1:1993 (Associação..., 1993).

During the determination of the basic specifications for a defibrillator and cardioverter analyzer it was determined that it should be able to test defibrillators that are not in compliance and that may deliver excessive energy, peak voltage or peak current or that may present a synchronism and charging time higher than prescribed. Table 1 shows a compilation of all specifications considered desirable in this study.

\section{Characteristics of the commercial defibrillator and cardioverter analyzers}

Table 2 shows a compilation of the specifications of several defibrillator and cardioverter analyzers currently available in the market, in order to facilitate the comparison between the minimum specifications desirable for this type of instrument.

\section{Results}

Analyzing the specifications listed in Table 2 it was observed that all of them presented problems, either because the specification of a certain parameter falls below the desired level for a defibrillator analyzer or because a certain feature was simply not specified. None of the analyzers that were studied provided a specification for environmental conditions complying with the requirements determined by this research $\left(0\right.$ to $40{ }^{\circ} \mathrm{C} / 30$ to $95 \%$ relative humidity).

\section{Discussion and Conclusion}

The uncertainty of the energy measurements for the analyzers QED 6, Impulse 7000 DP, Delta 3000-MKII, Delta 3000 and JAU-110 was in compliance with the requirement for maximum uncertainty in the measurement of energy, which was equal to $\pm 5 \%$ of the reading or $\pm 1,0 \mathrm{~J}$, whichever is greater. The uncertainties of measurement of peak voltage and peak current are not provided for the analyzers Delta 3000 and JM-01 according to the manufacturer website.

The uncertainty of measurement of charge time is not provided for the analyzers QED 6 and JM-01 according to the operation manual, and the analyzer Delta 3000 according to the manufacturer website. The measure is constituted by its estimation and uncertainty and, therefore, a result without its uncertainty is an incomplete result.

The maximum synchronization time that is measured by the analyzers JM-01 and JAU-110 is $100 \mathrm{~ms}$, shorter than the $120 \mathrm{~ms}$ suggested by this study. However, this value is 67\% higher than the limit for the delay time specified by the NBR IEC 60601-2-4:2005 (Associação..., 2005a) and the ANSI/AAMI DF80:2003 (American..., 2003) standards for cardioverters, so that does not necessarily imply a non compliance, only a criterion less rigid than the one adopted by this study. 


\section{Introdução}

A desfibrilação e a cardioversão elétrica são procedimentos terapêuticos comumente utilizados para a reversão de arritmias cardíacas graves, fazendo com que desfibriladores e cardioversores sejam de grande relevância na área clínica. Consequentemente, a correta e cuidadosa avaliação do desempenho destes equipamentos constitui uma exigência obrigatória para garantir tanto a segurança quanto a eficácia dessa categoria de equipamentos eletromédicos.

A avaliação de desfibriladores e cardioversores, tanto nas fases de projeto e desenvolvimento como nas fases de manutenção preventiva e corretiva, pode ser realizada experimentalmente por meio de equipamentos denominados analisadores de desfibriladores e cardioversores.

Nem sempre as exigências para o instrumento de medição utilizado na realização de um ensaio previsto por uma norma encontram-se bem detalhadas. Muitas vezes o ensaio descrito pela norma especifica o parâmetro a ser mensurado (e.g. o erro máximo admissível na leitura de tensão de um equipamento ensaiado), no entanto não especifica as características do instrumento de medição a ser utilizado para verificar a conformidade do equipamento ensaiado.

Para que um analisador de desfibriladores e cardioversores esteja apto a executar os ensaios previstos pelas normas ABNT NBR IEC 60601-1:1997 (Associação..., 1997), ABNT NBR IEC 60601-2-4:2005 (Associação..., 2005a) e ANSI/ AAMI DF80:2003 (American..., 2003) deve atender a vários quesitos, o que demanda uma especificação mínima desejável.

Esta pesquisa recomenda uma especificação mínima desejável de analisadores de desfibriladores e cardioversores, bem como para sistemas de análise de desfibriladores, cardioversores e esfigmomanômetros baseados em microcomputadores (Guedes e Moraes, 2001); avalia também se analisadores atualmente disponíveis comercialmente atendem a tais requisitos e se suas especificações são apresentadas ao usuário de modo adequado.

É importante enfatizar que o atendimento de avaliações envolvendo características metrológicas é mandatório neste assunto.

Adicionalmente, este artigo apresenta os conceitos de sistema de gestão de medição, instrumentos de medição e capacidade do processo. A apresentação destes conceitos e suas considerações têm o objetivo de auxiliar o leitor a estabelecer as especificações mínimas para outros instrumentos de medição fornecendo a base para determiná-las quando estas não são contidas na norma ou procedimento.
Medição é um processo de obtenção experimental de um ou mais valores que podem ser, razoavelmente, atribuídos a uma grandeza (Instituto..., 2009), sendo o sistema de gestão de medição o responsável pela comprovação metrológica e o controle contínuo dos processos de medição. Na seção de Sistemas de Gestão de Medição são apresentados os conceitos aplicados aos analisadores de desfibriladores e cardioversores e algumas normas que podem auxiliar na sua implementação e avaliação.

$\mathrm{Na}$ seção de Instrumentos de Medições são apresentadas algumas orientações para a escolha deste tipo de equipamento.

O estudo de capacidade de um processo pode auxiliar na determinação das especificações minimamente aceitáveis para um determinado instrumento de medição, sendo o cálculo do índice de capacidade uma das ferramentas que pode a ser utilizada para validar um processo.

\section{Materiais e Métodos}

\section{Sistema de gestão de medição}

Um sistema de medição é um conjunto de um ou mais instrumentos de medição e, frequentemente, outros dispositivos, compreendendo, se necessário, reagentes e insumos, montado e adaptado para fornecer informações destinadas à obtenção dos valores medidos, dentro de intervalos especificados para grandezas de tipos especificados (Instituto..., 2009). Além disso, um sistema de medição é uma ferramenta que possibilita conhecer, monitorar e controlar variáveis de qualquer processo, de acordo com especificações de trabalho predefinidas (Duarte Junior, 2008). Dessa forma, o objetivo de um sistema de gestão de medição (SGM) é gerenciar o risco de que os instrumentos e os processos de medição possam produzir resultados incorretos, afetando a qualidade dos produtos de uma organização (Associação..., 2004).

Para a norma ABNT NBR ISO 10012:2004, SGM é um "[...] conjunto de elementos interrelacionados e interativos, necessários para obter a comprovação metrológica e o controle contínuo dos processos de medição [...]" (Associação..., 2004, p. 1). Comprovação metrológica é, por sua vez, um “[...] conjunto de operações necessárias para assegurar que um equipamento de medição atenda aos requisitos do seu uso pretendido [...]" (Associação..., 2004, p. 2), o que envolve calibração, ajuste, reparos, identificação, lacre, verificação e documentação da adequação ao uso do instrumento (equipamento) de medição.

Os processos de medição visam comprovar que a grandeza específica em questão (mensurando) está 
em conformidade com os requisitos especificados. É necessário confiar nessas medições (confiabilidade metrológica) para se poder tomar decisões relacionadas aos produtos e processos em questão (Duarte Junior, 2008).

A norma ABNT NBR ISO/IEC 17025:2005 contém todos os requisitos que os laboratórios de ensaio e calibração devem atender se desejam demonstrar que têm implementado um sistema da qualidade, são tecnicamente competentes e que são capazes de produzir resultados tecnicamente válidos (Associação..., 2005b). Esta norma especifica os requisitos gerais para a competência em realizar ensaios e/ou calibrações, incluindo amostragem. Ela cobre ensaios e calibrações realizados, utilizando métodos normalizados, métodos não normalizados e métodos desenvolvidos pelo laboratório. Além disso, é uma norma aplicável a todas as organizações que realizam ensaios e/ou calibrações. Estas incluem, por exemplo, laboratórios onde o ensaio e/ou calibração são parte da inspeção e da certificação de produto (Associação..., 2005b). Portanto, seus requisitos devem ser considerados para a especificação de um sistema de medição.

\section{Instrumentos de medição}

Em um processo de calibração, é necessário determinar qual o instrumento de medição que melhor se adéqua a cada uma das grandezas a serem mensuradas. Esta é uma decisão que envolve um compromisso entre custo (do instrumento e do processo de medição e comprovação metrológica) e benefício (a exatidão/ precisão ou incerteza de medição obtida). Quando se trata do projeto ou aquisição de meios de medição, é necessário definir a adequação de um instrumento de medição com base em características como faixa de medição, resolução, classe ou incerteza declarada, uma vez que, via de regra, a verificação só será possível após o recebimento do instrumento (Duarte Junior, 2008).

Uma regra prática que pode ser utilizada neste caso é a "Regra do 4 a 10 vezes" que relaciona a resolução do instrumento com o erro máximo admissível do mensurando, onde de preferência a resolução deveria ser 1/10 do erro máximo admissível do mensurando, e no máximo 1/4 do mesmo. Em todos os casos, deve-se determinar um erro máximo admissível para o instrumento de medição que servirá de referência para a sua comprovação metrológica ou não (Duarte Junior, 2008).

Convém que o erro máximo admissível do instrumento de medição seja baseado no processo em que será utilizado. Quando aplicável, o erro deve estar em conformidade com as determinações das leis e ou das normas aplicáveis a estes sistemas de medição. A norma ABNT NBR ISO 10012-1:1993 orienta que o erro imputável à calibração deve ser tão pequeno quanto possível, e que, na maioria das áreas de medição, não deveria ser maior do que um terço e, de preferência, um décimo do erro máximo admissível do equipamento comprovado quando em uso (Associação..., 1993).

Outras condições, quando necessário, também devem ser especificadas, por exemplo, ambiente, capacitação do observador, método de medição (Duarte Junior, 2008).

Estas orientações visam minimizar os erros de leitura dos instrumentos, de forma que os valores lidos possam representar realmente as variações do mensurando, e não simplesmente incertezas de medição. Os métodos de medição e, em especial, os de calibração, deveriam ser baseados em normas ou procedimentos validados, e deveriam trazer informações inclusive sobre o correto uso, armazenamento e transporte de instrumentos de medição (Duarte Junior, 2008).

\section{Capabilidade ou capacidade}

De acordo com a norma ABNT NBR ISO 9000:2005, produto é definido como o resultado de um processo; capacidade é definida como a aptidão de uma organização, sistema ou processo de realizar um produto que irá atender aos requisitos (Associação..., 2005c).

O estudo de capacidade visa definir se um processo de comportamento conhecido é capaz de produzir itens ou prestar serviços conforme as especificações estabelecidas (Moraes, 2006).

Um processo é dito capaz se sua variabilidade total (inerente ou natural) é uma pequena parcela do erro máximo admissível ao produto. Portanto, um processo de calibração de um produto é dito capaz se a incerteza de medição expandida do processo de calibração for igual a uma pequena parcela do erro máximo admissível para aquele produto.

Os termos capacidade, capabilidade, aptidão e desempenho de processo possuem o mesmo significado, segundo Toledo (2005), pois todos representam medidas estatísticas que indicam a taxa de variação existente no processo em relação às especificações dos produtos.

A avaliação da capacidade do processo costuma ser realizada mediante o cálculo de índices de capacidade. Os limites de especificação são externos ao processo. Eles representam os requisitos de engenharia que satisfazem um projeto. Estes requisitos podem ser determinados para atender necessidades técnicas, de clientes ou outras. 
Os limites de controle são internos ao processo, eles representam a sua dispersão e refletem a faixa esperada de variação do processo.

O índice de capacidade é a relação entre os limites de especificação e limites de controle, conforme apresenta a Equação 1 (Samohyl, 2005).

$$
C=\frac{L E S-L E I}{L C S-L C I}
$$

na qual $L E S$ e $L E I$ são os limites de especificação superior e inferior, e $L C S$ e $L C I$ são os limites de controle superior e inferior, respectivamente.

Para os processos que produzem uma distribuição normal, o índice capacidade potencial $C_{p}$ fornece uma medida indireta da habilidade potencial do processo em atender às especificações da característica da qualidade de interesse, considerando o processo centrado na média das especificações. O índice $C_{p}$ é obtido pela Equação 2 (Moraes, 2006).

$$
C_{p}=\frac{L E S-L E I}{6 \cdot \hat{\sigma}}
$$

na qual $L E S$ e $L E I$ são os limites de especificação superior e inferior, respectivamente, e ô é a estimativa do desvio padrão do processo.

Assim, para o processo ser considerado capaz, o índice $C_{p}$ deve $\geq 1$, o que equivale a dizer que pelo menos $99,73 \%$ dos produtos serão conformes (Samohyl, 2005). A capacidade de um sistema de medição pode ser utilizada para a avaliação do processo de medição.

\section{Especificações básicas de um analisador de desfibriladores e cardioversores}

A determinação das especificações básicas de um analisador de desfibriladores e cardioversores foi obtida a partir dos requisitos para desfibriladores e cardioversores determinados pelas normas NBR IEC 60601-2-4:2005 (Associação..., 2005a) e ANSI/AAMI DF80:2003 (American..., 2003). Uma compilação dos requisitos considerados é apresentada na Tabela 1.

Um analisador de desfibriladores deve estar apto a realizar os ensaios prescritos pelas normas mencionadas acima nas condições de operação previstas: temperatura de 0 a $40^{\circ} \mathrm{C}$, umidade relativa entre 30 e $95 \%$ sem condensação.

As normas NBR IEC 60601-2-4 (Associação..., 2005a) e ANSI/AAMI DF80 (American... e Association..., 2003) estabelecem que a máxima energia de descarga selecionável por um desfibrilador não deve exceder $360 \mathrm{~J}$. Estas normas também estabelecem que a energia entregue não deve variar por mais que $\pm 15 \%$ da energia selecionada, ou $\pm 3 \mathrm{~J}$ (maior valor entre ambos). Portanto, um desfibrilador ainda estaria em conformidade com essas normas caso apresentasse uma descarga com energia entregue igual a $414 \mathrm{~J}$ quando a energia selecionada fosse $360 \mathrm{~J}$.

Considerando que um analisador avalie desfibriladores que estejam operando fora de suas especificações, apresentando energias excessivas de saída, arbitrou-se que o analisador deva medir desvios de até 50\% na máxima energia entregue para cobrir os piores casos. Assim, um analisador que esteja apto a medir, ou pelo menos suportar, energias de até $621 \mathrm{~J}$ atenderia adequadamente as necessidades encontradas.

A norma ANSI/AAMI DF80:2003 é uma revisão conjunta das normas ANSI/AAMI DF2:1996 e ANSI/ AAMI DF39:1993 (American...,1993). A norma ANSI/ AAMI DF80 não estabelece o erro máximo admissível para os instrumentos de medição utilizados na realização dos ensaios por ela estabelecidos. Entretanto, a norma ANSI/AAMI DF2, na qual ela foi baseada, estabelece que todos esses instrumentos de ensaio devem ter a maior exatidão possível, e não mais que um terço do erro máximo admissível da grandeza a ser medida ou $\pm 5 \%$ da variável a ser medida em que apenas limites máximos e mínimos são fornecidos. Portanto, tem-se que a incerteza máxima aceitável de um analisador para as medidas de energia é igual a $\pm 5 \%$ ou $\pm 1,0 \mathrm{~J}$, o que for maior dentre os dois.

Estas considerações estão de acordo com aquelas apontadas como desejáveis para um instrumento de medição e as orientações da norma ABNT NBR ISO 10012-1 (Associação..., 1993).

De acordo com a "Regra do 4 a 10 vezes" e considerando que o erro máximo admissível para o valor da energia entregue quando comparado ao valor da energia selecionada é igual a $\pm 3 \mathrm{~J}$, de acordo com as normas NBR IEC 60601-2-4 (Associação..., 2005a) e ANSI/AAMI DF80 (American..., 2003), a resolução de um analisador deve ser de pelo menos $0,75 \mathrm{~J}$.

A norma ANSI/AAMI DF80:2003 (American..., 2003) estabelece um limite para a corrente de pico de 66 A para descarga senoidal amortecida com uma energia selecionada igual a $360 \mathrm{~J}$, e de 40 A para descarga exponencial truncada, quando realizadas sobre uma resistência de $50 \Omega$. Conforme mencionado anteriormente, considerando que um analisador deve estar apto a ensaiar desfibriladores não conformes, os quais podem vir a apresentar picos de corrente superior aos limites estabelecidos para descargas efetuadas sobre uma resistência de carga de $50 \Omega$, arbitrou-se que um desvio de $50 \%$ do limite estabelecido por esta norma deve cobrir grande parte dos casos, de modo que um analisador capaz de medir, ou pelo menos suportar, correntes de pico de até 99 A atenderia adequadamente as necessidades encontradas. 
Tabela 1. Prescrições para desfibriladores e cardioversores determinadas pelas normas ABNT NBR IEC 60601-2-4:2005 e ANSI/ AAMI DF80:2003.

Table 1. Requirements for Defibrillators and Cardioverters from ABNT NBR IEC 60601-2-4:2005 and ANSI/AAMI DF80:2003 standards.

\begin{tabular}{|c|c|c|c|}
\hline \multicolumn{2}{|r|}{ Norma } & \multirow{2}{*}{$\begin{array}{c}\text { ABNT NBR IEC 60601-2-4:2005 } \\
0 \text { a } 40{ }^{\circ} \mathrm{C} \text { (para uso em salas médicas) }\end{array}$} & \multirow{2}{*}{$\frac{\text { ANSI/AAMI DF80:2003 }}{0 \text { a } 40^{\circ} \mathrm{C}}$} \\
\hline & Temperatura & & \\
\hline operação & Umidade relativa do ar & $\begin{array}{c}30 \text { a } 95 \% \text { (sem condensação) (para uso } \\
\text { em salas médicas) }\end{array}$ & 30 a 95\% (sem condensação) \\
\hline \multirow{2}{*}{$\begin{array}{c}\text { Máxima energia } \\
\text { selecionável }\end{array}$} & Desfibrilações externas & $360 \mathrm{~J}$ & $360 \mathrm{~J}$ \\
\hline & Desfibrilações internas & $50 \mathrm{~J}$ & $50 \mathrm{~J}$ \\
\hline \multicolumn{2}{|c|}{ Exatidão da energia fornecida } & $\begin{array}{l} \pm 15 \% \text { da energia selecionada ou } \pm 3 \mathrm{~J} \\
\text { o que for maior dentre os dois. }\end{array}$ & $\begin{array}{c} \pm 15 \% \text { da energia selecionada ou } \pm 3 \mathrm{~J} \\
\text { o que for maior dentre os dois. }\end{array}$ \\
\hline \multirow{2}{*}{$\begin{array}{l}\text { Tempo de } \\
\text { sincronismo }\end{array}$} & Sinal ECG & $60 \mathrm{~ms}$ & $60 \mathrm{~ms}$ \\
\hline & Sinal de sincronismo & $25 \mathrm{~ms}$ & $25 \mathrm{~ms}$ \\
\hline \multicolumn{2}{|c|}{ Corrente de pico máxima } & ------------ & $\begin{array}{l}\text { (Referência à norma ANSI/AAMI } \\
\text { DF2:1996) } \\
\text { Para descarga senoidal amortecida: } \\
\text { - 96 A (Resistência de carga }=25 \Omega \text { ), } \\
\text { - 66 A (Resistência de carga }=50 \Omega \text { ), } \\
\text { - 46 A (Resistência de carga }=100 \Omega \\
\text { ou } 125 \Omega \text { ). } \\
\text { Para descarga exponencial truncada: } \\
\text { - 80 A (Resistência de carga }=25 \Omega \text { ) } \\
\text { - 40 A (Resistência de carga }=50 \Omega \text { ). } \\
\text { - 20 A (Resistência de carga }=100 \Omega \\
\text { ou } 125 \Omega \text { ) }\end{array}$ \\
\hline \multicolumn{2}{|c|}{ Tensão de pico máxima } & $5000 \mathrm{~V}$ (resistência de carga $=175 \Omega$ ) & $5.000 \mathrm{~V}$ (resistência de carga $=175 \Omega$ ) \\
\hline \multirow{4}{*}{ Tempo de carga } & $\begin{array}{c}\text { Desfibriladores manuais } \\
\text { (uso frequente) }\end{array}$ & $15 \mathrm{~s}$ & $15 \mathrm{~s}$ \\
\hline & $\begin{array}{l}\text { Desfibriladores manuais } \\
\text { (uso não frequente) }\end{array}$ & $\begin{array}{c}20 \mathrm{~s} \\
25 \mathrm{~s} \text { (bateria depreciada por } \\
15 \text { descargas de energia máxima) }\end{array}$ & $\begin{array}{c}20 \mathrm{~s} \\
25 \mathrm{~s} \text { (bateria depreciada por } \\
15 \text { descargas de energia máxima) }\end{array}$ \\
\hline & DEA (uso frequente) & $\begin{array}{c}30 \mathrm{~s} \\
40 \mathrm{~s} \text { (ao ligar o desfibrilador) }\end{array}$ & $\begin{array}{c}30 \mathrm{~s} \\
40 \mathrm{~s} \text { (ao ligar o desfibrilador) }\end{array}$ \\
\hline & DEA (uso não frequente) & $\begin{array}{l}\text { 35 s } \\
40 \mathrm{~s} \text { (bateria depreciada por } \\
15 \text { descargas de energia máxima) } \\
45 \mathrm{~s} \text { (ao ligar o desfibrilador) } \\
50 \mathrm{~s} \text { (ao ligar o desfibrilador } \\
\text { após a bateria ser depreciada por } \\
15 \text { descargas de energia máxima) }\end{array}$ & $\begin{array}{l}\text { 35 s } \\
40 \mathrm{~s} \text { (bateria depreciada por } \\
15 \text { descargas de energia máxima) } \\
45 \mathrm{~s} \text { (ao ligar o desfibrilador) } \\
50 \mathrm{~s} \text { (ao ligar o desfibrilador } \\
\text { após a bateria ser depreciada por } \\
15 \text { descargas de energia máxima) }\end{array}$ \\
\hline \multirow[t]{2}{*}{ Largura do pulso } & $\begin{array}{l}\text { Onda senoidal } \\
\text { amortecida } \\
\left(t_{10}-\text { intervalo de tempo }\right. \\
\text { no qual a corrente do } \\
\text { primeiro lóbulo da onda } \\
\text { desfibrilatória é superior } \\
\text { a } 10 \% \text { da corrente de } \\
\text { pico desta onda })\end{array}$ & & $3,1 \mathrm{~ms} \leq t_{10} \leq 9,2 \mathrm{~ms}$ \\
\hline & $\begin{array}{l}\text { Onda exponencial } \\
\text { truncada } \\
\left(t_{d}-\text { largura da forma de }\right. \\
\text { onda de uma descarga } \\
\text { exponencial truncada })\end{array}$ & ---- & $3 \mathrm{~ms} \leq t_{d} \leq 24 \mathrm{~ms}$ \\
\hline
\end{tabular}


As normas NBR IEC 60601-2-4 (Associação..., 2005a) e ANSI/AAMI DF80 (American..., 2003) estabelecem que a tensão de saída da descarga de um desfibrilador efetuada sobre uma resistência de carga de $175 \Omega$ não deve exceder $5 \mathrm{kV}$, de modo que um analisador deve estar apto a medir tensões de pico de até $5.000 \mathrm{~V}$. Este valor já apresenta uma margem de segurança quando se considera a utilização de resistências de carga inferiores a $175 \Omega$.

Como apenas limites máximos e mínimos para a corrente de pico de uma descarga são especificados, tem-se que a norma ANSI/AAMI DF2:1996 (American..., 1996) estabelece uma incerteza máxima de $5 \%$ para a medição de corrente. Portanto, a incerteza máxima aceitável para as medições de corrente de um analisador é igual a 5\%.

Considerando os limites para corrente de pico de descargas senoidais amortecidas estabelecidos pela norma ANSI/AAMI DF80 (American..., 2003) e que as energias selecionadas para um desfibrilador podem ser tão pequenas quanto $1 \mathrm{~J}$, tem-se que a corrente de pico de uma descarga senoidal pode ser de apenas 2,37 A, quando realizada sobre uma resistência de $50 \Omega$, o que também implica em uma tensão de pico de apenas 118,5 V. Como as incertezas para a corrente de pico e tensão de pico são de $\pm 5 \%$, de acordo com a "Regra do 4 a 10 vezes" tem-se que a resolução mínima para a corrente de pico para um analisador deve ser igual a $0,03 \mathrm{~A}$ e a resolução mínima para a tensão de pico para um analisador deve ser igual a $1,5 \mathrm{~V}$.

Como a tensão de pico mínima prevista para uma descarga é de $118,5 \mathrm{~V}$, considera-se que o nível do disparador (trigger) (tensão mínima de pico para que o analisador detecte a descarga) deve ser inferior a $100 \mathrm{~V}$.

$\mathrm{O}$ analisador deve também medir o tempo de sincronismo (tempo de atraso decorrente desde a última onda R do sinal de ECG gerado pelo analisador até o pico do pulso desfibrilatório). Tanto a norma NBR IEC 60601-2-4:2005 quanto a norma ANSI/ AAMI DF80:2003 determinam que, em descargas sincronizadas, o pico do pulso desfibrilatório deve ocorrer dentro de um intervalo de $60 \mathrm{~ms}$ do pico da onda R do ECG. Para o caso da descarga sincronizada utilizar um sinal de sincronismo externo, como por exemplo, a saída de sincronismo de um monitor cardíaco, estas normas determinam que o pico do pulso desfibrilatório deve ocorrer dentro de um intervalo de $25 \mathrm{~ms}$ da aplicação do último sinal de sincronismo.

$\mathrm{O}$ analisador deve estar apto a medir o tempo de sincronismo de um cardioversor não conforme, que apresente um tempo de sincronismo superior ao limite estabelecido pelas normas. Arbitrou-se como limite mínimo para a faixa de medição o dobro do intervalo considerado como limite pelas normas, de modo que esta deve compreender pelo menos o intervalo de 0 a $120 \mathrm{~ms}$.

Assim como o especificado para a corrente de pico, pelo fato das normas estabelecerem apenas limites máximos para o tempo de sincronismo, tem-se que a incerteza máxima aceitável para as medições do tempo de sincronismo de um analisador deve ser igual a 5\%.

A norma ANSI/AAMI DF2 (American..., 1996) estabelece que o sinal de ECG utilizado para o sincronismo deve ter amplitude de $1 \mathrm{~m} \mathrm{~V}_{\mathrm{pp}}$ e frequência de 60 a 150 BPM; portanto, o analisador deve gerar um sinal de ECG que atenda estas especificações.

Para uma análise mais completa do desempenho de desfibriladores e cardioversores sob ensaio é desejável que o analisador também seja capaz de determinar os tempos de carga de um desfibrilador. Para desfibriladores externos automáticos (DEAs) de utilização não frequente, alimentado por uma bateria depreciada por 15 descargas de máxima energia, as normas NBR IEC 60601-2-4 (Associação..., 2005a) e ANSI/AAMI DF80 (American..., 2003) estabelecem um tempo máximo de $50 \mathrm{~s}$, considerando que neste intervalo já estão inclusos os intervalos de tempo relativos ao auto-teste, análise do ECG e carga.

Analogamente ao considerado para a faixa de medição do tempo de sincronismo, arbitrou-se que a faixa de medição do analisador para o tempo de carga deve ser pelo menos duas vezes o limite estabelecido pelas normas, de modo que a faixa de medição do analisar deve ser de no mínimo $100 \mathrm{~s}$.

Assim como o especificado para a corrente de pico, pelo fato das normas estabelecerem apenas limites máximos para o tempo de sincronismo e para os tempos de carga, tem-se que a incerteza máxima do analisador na medição destes parâmetros deve ser de $5 \%$.

As normas ANSI/AAMI DF2 (American..., 1996) e ANSI/AAMI DF80 (American..., 2003) determinam parâmetros para descarga senoidal amortecida e exponencial truncada. É desejável que o analisador seja capaz de realizar a medição destes parâmetros. Dentre os parâmetros para descargas senoidal amortecida em uma impedância de $50 \Omega$, destaca-se $t_{r}$ (tempo de subida do primeiro lóbulo) que deve estar entre 0,40 e $1,42 \mathrm{~ms} ; t_{10}$ (tempo no qual a corrente do primeiro lóbulo da onda desfibrilatória é superior a $10 \%$ da corrente de pico) que deve estar entre 3,1 e 9,2 ms; $t_{50}$ (tempo no qual a corrente do primeiro lóbulo da onda desfibrilatória é superior a $50 \%$ da corrente de pico) que deve estar entre 2,3 e 6,4 ms.

Dentre os parâmetros para descargas exponencial truncada em uma impedância de $50 \Omega$, destaca-se $t_{d}$ (largura do pulso) que deve estar entre 3 e 24 ms; 
tilt (relação entre a corrente inicial e final da forma de onda) que deve ser de no máximo igual a $80 \%$.

A Tabela 2 compila as especificações estabelecidas como desejáveis por esta pesquisa.

\section{Resultados}

\section{Características dos analisadores de desfibriladores e cardioversores comerciais}

A Tabela 2 apresenta também a compilação das especificações de diversos analisadores de desfibriladores e cardioversores disponíveis comercialmente e as exigências mínimas desejadas para este tipo de instrumento de acordo com esta pesquisa. Todas as informações foram obtidas dos manuais de operação dos analisadores e dos websites dos fabricantes.

\section{Discussão}

A seção "Instrumentos de Medição" orienta que o erro imputável à calibração deve ser tão pequeno quanto possível, e que, na maioria das áreas de medição, não deveria ser maior do que um terço e, de preferência, um décimo do erro máximo admissível do equipamento comprovado quando em uso (Associação..., 1993).

Na seção "Capacidade ou Capabilidade" deste artigo, a partir da Equação 2 e da análise da capacidade de um processo onde se considera que a incerteza padrão de um instrumento determina os seus limites de especificação superior e inferior $L E S$ e $L I S$, pode-se observar que o processo seria considerado capaz $\left(C_{p} \geq 1\right)$ quando a estimativa do desvio padrão do processo $\hat{\sigma}$ fosse menor ou igual a um terço do desvio padrão do instrumento.

Conforme mencionado anteriormente, na seção "Especificações básicas" de um analisador de desfibriladores e cardioversores, a norma ANSI/ AAMI DF2 estabelece que todos os instrumentos e equipamentos de ensaio utilizados na realização dos ensaios por ela especificados devem ter a maior exatidão possível, e não mais que um terço do erro máximo admissível da grandeza a ser medida. Observa-se, portanto, que as orientações e conceitos apresentados nas seções "Instrumentos de Medição" e "Capabilidade ou Capacidade" estão alinhados com a norma em questão.

Estas orientações e conceitos podem ser utilizados como base para a determinação e avaliação de especificações, quando estas não se encontram especificadas por uma norma ou procedimento, auxiliando o leitor na determinação de especificações mínimas para outros instrumentos de medição.
Uma análise das especificações declaradas para os analisadores citados na Tabela 2 permite concluir que todos apresentam problemas nas suas especificações, ou que a especificação de um determinado parâmetro está aquém do desejado para um analisador de desfibriladores e cardioversores, ou devido à ausência da especificação de uma certa característica.

O erro máximo da medição de energia especificado para os analisadores Fluke QED 6 e Impulse, Delta 7000 DP, MTK-Biomed 3000-MKII, NETECH Delta 3000, e Ourox JAU 110 atendem integralmente o requisito de erro máximo admissível permitido para a medição de energia, que é igual a $\pm 5 \%$ da leitura ou $\pm 1,0 \mathrm{~J}$, o maior valor dentre os dois, conforme discutido acima. $\mathrm{O}$ erro máximo das medições de energia realizadas pelo analisador Transmai JM-01 não é especificado no manual de operação do analisador.

A faixa de medição da energia do analisador MTK-Biomed Delta 3000 MKII vai até 400,0 J, o que não é apenas inferior à especificação desejável por esta pesquisa, mas também é inferior à máxima energia que um desfibrilador pode vir a apresentar quando uma energia igual a $360 \mathrm{~J}$ é selecionada. A faixa de medição da energia do analisador Fluke Impulse 7000 vai até $600 \mathrm{~J}$, o que equivale a uma energia $44,9 \%$ superior à máxima energia considerada como conforme para uma energia selecionada igual a $360 \mathrm{~J}$, bem próxima dos $50 \%$ a mais $(621 \mathrm{~J})$ sugeridos por esta pesquisa. As faixas de medição dos demais analisadores atendem integralmente à especificação para a faixa sugerida.

A resolução apresentada para as medições de energia por todos os analisadores pesquisados, assim como o nível do disparador (trigger) atenderam as exigências tidas como desejáveis neste estudo.

Nenhum dos analisadores pesquisados apresenta uma especificação para a condição ambiental que atendesse integralmente às exigências determinadas pelas normas NBR IEC 60601-2-4 (Associação..., 2005a) e ANSI/ AAMI DF80 (American..., 2003). A especificação relativa à temperatura de operação apresentada pelo analisador Fluke QED 6 está condizente com a determinada pela norma NBR IEC 60601-2-4 (Associação..., 2005a) $\left(0\right.$ a $\left.40{ }^{\circ} \mathrm{C}\right)$. Os analisadores MTK-Biomed Delta 3000 MKII $\left(15\right.$ a $\left.40^{\circ} \mathrm{C}\right)$ e o Fluke Impulse $7000\left(10 \mathrm{a} 40^{\circ} \mathrm{C}\right)$ não atendem às exigências das normas 60601-2-4 (Associação..., 2005a) nem da ANSI/AAMI DF80 (American..., 2003). Os analisadores NETECH Delta 3000, Transmai JM-01 e Ourox JAU 110 não especificam as temperaturas de operação.

Nenhum dos analisadores apresentou uma especificação de faixa de operação para a umidade relativa do ar que atendesse às exigências desejáveis, limitando-se, quando especificado, a uma umidade 


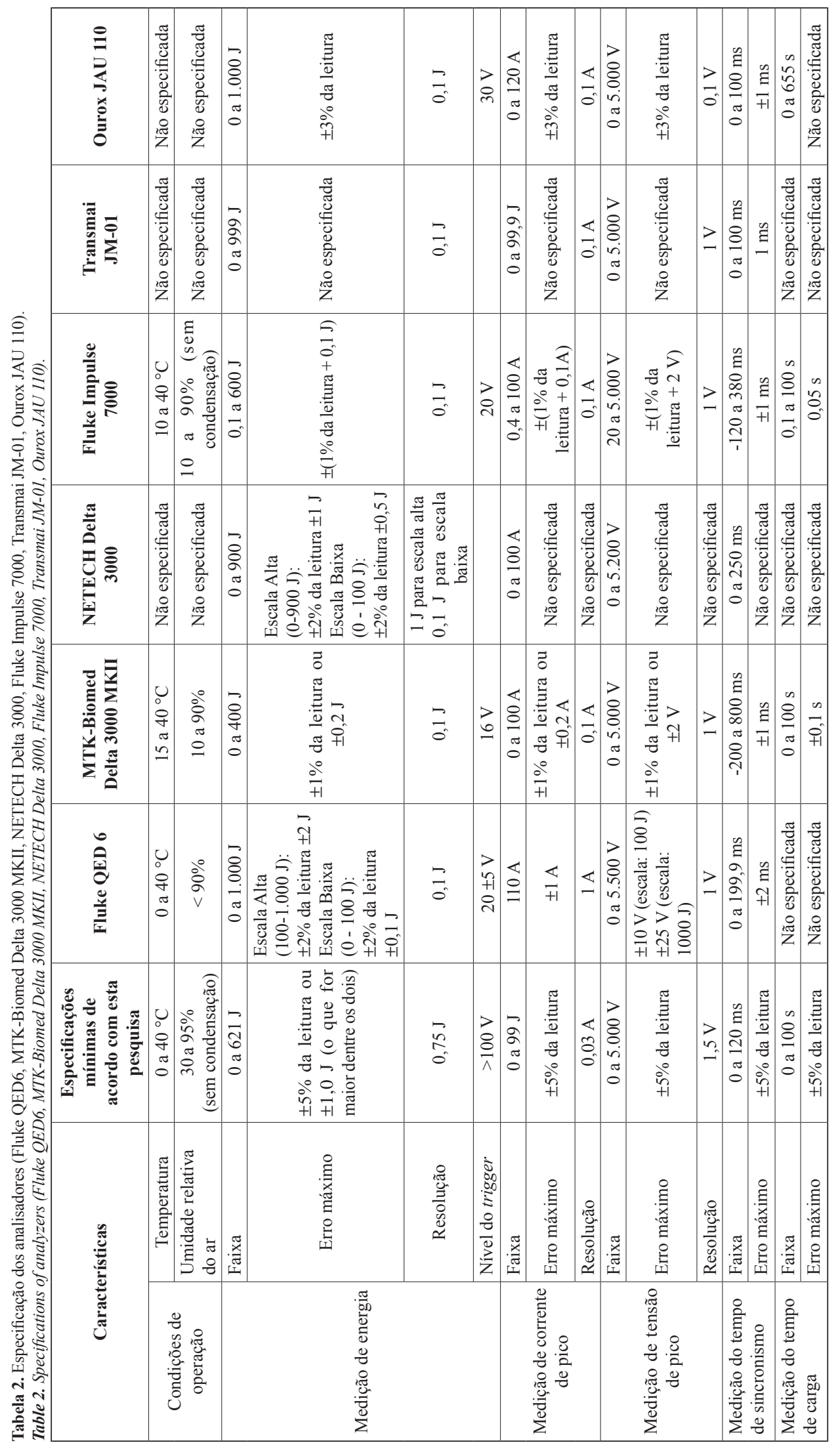


relativa máxima de $90 \%$, enquanto a determinação das normas 60601-2-4 (Associação..., 2005a) e ANSI/ AAMI DF80 (American..., 2003) é igual a 95\%.

As especificações relativas às medições de corrente e tensão de pico na maioria dos analisadores se limitaram a determinar a corrente e tensão máxima que eles estariam aptos a medir; neste quesito todos os analisadores pesquisados atenderam aos requisitos tidos como desejáveis por esta pesquisa. Entretanto, especificações importantes como a resolução das medições, precisão e erro máximo da medição não são apresentados pelos analisadores NETECH Delta 3000 e Transmai JM-01. O fato de não apresentarem o erro máximo dos valores medidos de corrente e tensão de pico torna os seus resultados incompletos, visto que toda medida é formada pela sua estimativa juntamente com sua incerteza.

A janela de medição para o tempo de sincronismo dos analisadores Transmai JM-01 e Ourox JAU-110 é igual a $100 \mathrm{~ms}$, portanto menor que os $120 \mathrm{~ms}$ especificados como desejado por esta pesquisa. Entretanto, este valor é $67 \%$ superior ao limite para o tempo de atraso especificado para os cardioversores pelas normas 60601-2-4:2005 (Associação..., 2005a) e ANSI/AAMI DF80:2003 (American..., 2003), não implicando necessariamente em uma não conformidade, apenas uma consideração menos rígida que a adotada neste estudo. As janelas de medição do tempo de atraso dos demais analisadores foram todas superiores ao considerado como desejável. Já as especificações relativas aos erros máximos da medição deste parâmetro, que de acordo com a norma ANSI/AAMI DF2:1996 (American..., 2003) deve ser de no máximo $5 \%$, é igual a \pm 1 ms para os analisadores MTK-Biomed Delta 3000 MKII, Fluke Impulse 7000 e Ourox JAU-110. Há, portanto, atendimento à exigência apenas para medições iguais ou superiores a $20 \mathrm{~ms}$, valor este que não deve ser considerado como pequeno, o que torna a incerteza aquém das expectativas.

$\mathrm{O}$ analisador Fluke QED6 especifica um erro máximo para as medições do tempo de atraso igual a $\pm 2 \mathrm{~ms}$, o que torna a incerteza de suas medições fora do limite considerado como desejável para medições inferiores a $40 \mathrm{~ms}$, o que é bastante comum para uma grande quantidade de cardioversores atualmente comercializados. O portal da NETECH não fornece dados relativos ao erro máximo das medições do tempo de atraso do seu analisador modelo Delta 3000.

As especificações relativas às janelas de medição do tempo de carga de todos os analisadores foram superiores à exigência determinada nesta pesquisa. Entretanto, os analisadores Fluke QED6, NETECH Delta 3000, Transmai JM-01 e Ourox JAU-110 não definem os erros máximos de suas medições. Os analisadores Fluke Impulse 7000 e MTK-Biomed Delta 3000 MKII especificam um erro máximo nas medições de tempo de carga iguais a $\pm 0,05 \mathrm{e} \pm 0,1 \mathrm{~s}$, respectivamente. Estes valores são iguais ao limite de $\pm 5 \%$ da leitura para medições iguais a 1 e $2 \mathrm{~s}$, atendendo satisfatoriamente às exigências, visto que estes são valores muito pequenos para o tempo de carga.

Dentre os analisadores pesquisados, apenas o analisador Fluke Impulse 7000 realiza a medição dos parâmetros $t_{r}$ (tempo de subida do primeiro lóbulo), $t_{10}$ (tempo no qual a corrente do primeiro lóbulo da onda desfibrilatória é superior a $10 \%$ da corrente de pico) e $t_{50}$ (tempo no qual a corrente do primeiro lóbulo da onda desfibrilatória é superior a $50 \%$ da corrente de pico) em descargas senoidal amortecida. Este analisador é também o único que realiza a medição dos parâmetros $t_{d}$ (largura do pulso) e tilt em descarga exponencial truncada.

\section{Conclusão}

Ao avaliarmos as especificações destacadas na Tabela 2, observamos que todos os dispositivos estudados apresentaram problemas, seja por não atenderem a determinado parâmetro ou porque determinada característica simplesmente não fora especificada.

É importante salientar que a medição de qualquer um dos parâmetros destacados neste artigo não tem validade legal se a calibração deles não for comprovada, sendo muito importante a determinação de um procedimento de calibração adequado.

Finalmente, os autores consideram que a proposta inserida neste artigo deverá ser continuamente aprimorada e atualizada conforme o avanço científico/ tecnológico. Um exemplo a ser analisado futuramente corresponderia à necessidade de se aprimorar as folhas de dados dos equipamentos por parte dos fabricantes, tanto em seus websites como nos manuais de instruções e outros documentos acompanhantes.

\section{Referências}

American National Standards Institute - ANSI, Association for the Advancement of Medical Instrumentation - AAMI. DF39: Automatic, External Defibrillators and Remote Control Defibrillators. ANSI; 1993.

American National Standards Institute - ANSI, Association for the Advancement of Medical Instrumentation- AAMI. DF2: Cardiac Defibrillator Devices. ANSI; 1996.

American National Standards Institute - ANSI, Association for the Advancement of Medical Instrumentation - AAMI. DF80: Medical electrical equipment - Part 2-4: Particular requirements for the safety of cardiac defibrillators (including automated external defibrillators). ANSI; 2003. 
Associação Brasileira de Normas Técnicas - ABNT. NBR ISO 10012-1: Requisitos para garantia da qualidade para equipamento de medição. Rio de Janeiro: ABNT; 1993.

Associação Brasileira de Normas Técnicas -ABNT. NBR IEC 60601-1: Equipamento eletromédico Parte 1 - Prescrições para segurança. Rio de Janeiro: ABNT; 1997.

Associação Brasileira de Normas Técnicas - ABNT. NBR ISO 10012: Sistema de gestão de medição - Requisitos para os processos de medição e equipamento de medição. Rio de Janeiro: ABNT; 2004.

Associação Brasileira de Normas Técnicas - ABNT. NBR IEC 60601-2-4: Equipamento eletromédico - Parte 2-4: Prescrições particulares para segurança de desfibriladores cardíacos. Rio de Janeiro: ABNT; 2005a.

Associação Brasileira de Normas Técnicas - ABNT. NBR ISO/IEC 17025: Requisitos gerais para a competência de laboratórios de ensaio e calibração. Rio de Janeiro: ABNT; 2005b.

Associação Brasileira de Normas Técnicas - ABNT. NBR ISO 9000: Sistemas de gestão da qualidade - Fundamentos e vocabulário. Rio de Janeiro: ABNT; 2005c.
Duarte Junior NSF. Sistema de gestão de medição: importante, mas nem sempre reconhecido. Revista Metrologia \& Instrumentação. 2008; 7(56). Disponível em: http:// latoqualitas.com.br/artigos/Artigo_9.pdf

Guedes JR, Moraes R. Sistema para a análise de desfibriladores, cardioversores e esfigmomanômetros baseado em um microcomputador. Revista Brasileira de Engenharia Biomédica. 2001; 17(1):3-12.

Instituto Nacional de Metrologia, Qualidade e Tecnologia - INMETRO. Vocabulário Internacional de Metrologia: Conceitos Fundamentais e Gerais e Termos Associados (VIM 2008). Rio de Janeiro: INMETRO; 2009.

Moraes CF. Estudo da utilização do gráfico de controle individual e do índice de capabilidade sigma para dados não normais [dissertação]. Itajubá: Universidade Federal de Itajubá; 2006.

Samohyl RW. Controle estatístico de processo e ferramentas da qualidade. In: Carvalho MM, Paladini EP, editors. Gestão da qualidade: teoria e casos. Rio de Janeiro: Elsevier; 2005.

Toledo TPA. Uma investigação sobre índices de capabilidade com ênfase na metodologia Seis Sigma [dissertação]. Itajubá: Universidade Federal de Itajubá; 2005.

\section{Autores}

